\title{
A compendium of DIS3 mutations and associated transcriptional signatures in plasma cell dyscrasias
}

\author{
Marta Lionetti ${ }^{1,2 *}$, Marzia Barbieri ${ }^{2 *}$, Katia Todoerti $^{3}$, Luca Agnelli ${ }^{1,2}$, \\ Sonia Fabris ${ }^{2}$, Giovanni Tonon ${ }^{4}$, Simona Segalla ${ }^{4}$, Ingrid Cifola ${ }^{5}$, Eva Pinatel ${ }^{5}$, \\ Pierfrancesco Tassone ${ }^{6}$, Pellegrino Musto ${ }^{3}$, Luca Baldini ${ }^{1,2}$ and Antonino Neri ${ }^{1,2}$ \\ ${ }^{1}$ Department of Clinical Sciences and Community Health, University of Milano, Milan, Italy \\ ${ }^{2}$ Hematology Unit, Fondazione IRCCS Ca' Granda, Ospedale Maggiore Policlinico, Milan, Italy \\ ${ }^{3}$ Laboratory of Pre-Clinical and Translational Research, IRCCS-CROB, Referral Cancer Center of Basilicata, Rionero in \\ Vulture (PZ), Italy \\ ${ }^{4}$ Functional Genomics of Cancer Unit, Division of Experimental Oncology, IRCCS San Raffaele Scientific Institute, Milan, Italy \\ ${ }^{5}$ Institute for Biomedical Technologies, National Research Council, Milan, Italy \\ ${ }^{6}$ Department of Experimental and Clinical Medicine, Magna Graecia University, Catanzaro, Italy \\ *These authors have contributed equally to this work
}

Correspondence to:

Antonino Neri, e-mail: antonino.neri@unimi.it

Marta Lionetti, e-mail: marta.lionetti@unimi.it

Keywords: multiple myeloma, plasma cell leukemia, DIS3, next-generation sequencing

Received: May 08, $2015 \quad$ Accepted: July 06, 2015

Published: July 20, 2015

\section{ABSTRACT}

DIS3 is a catalytic subunit of the human exosome complex, containing exonucleolytic (RNB) and endonucleolytic (PIN) domains, recently found mutated in multiple myeloma (MM), a clinically and genetically heterogeneous form of plasma cell (PC) dyscrasia. We analyzed by next-generation sequencing (NGS) the DIS3 PIN and RNB domains in purified bone marrow PCs from 164 representative patients, including 130 cases with MM, 24 with primary PC leukemia and 10 with secondary PC leukemia. DIS3 mutations were found respectively in $18.5 \%, 25 \%$ and $30 \%$ of cases. Identified variants were predominantly missense mutations localized in the RNB domain, and were often detected at low allele frequency. DIS3 mutations were preferentially carried by IGH-translocated/nonhyperdiploid patients. Sequential analysis at diagnosis and relapse in a subset of cases highlighted some instances of increasing DIS3 mutation burden during disease progression. NGS also revealed that the majority of DIS3 variants in mutated cases were comparably detectable at transcriptional level. Furthermore, gene expression profiling analysis in DIS3-mutated patients identified a transcriptional signature suggestive for impaired RNA exosome function. In conclusion, these data further support the pathological relevance of DIS3 mutations in plasma cell dyscrasias and suggest that DIS3 may represent a potential tumor suppressor gene in such disorders.

\section{INTRODUCTION}

Multiple myeloma (MM) is a fatal malignancy of bone marrow (BM) plasma cells (PCs) whose pathogenesis is only partially understood. PC dyscrasias show a wide clinical presentation ranging from the presumed pre-malignant condition of monoclonal gammopathy of undetermined significance (MGUS) to smoldering MM
(SMM), symptomatic MM, and extra-medullary MM or plasma cell leukemia (PCL) [1]. PCL can be distinguished into primary PCL (pPCL), originating de novo without any prior history of MM, or secondary PCL (sPCL), arising from a preexisting myeloma tumor that eventually progressed to the leukemic phase [2].

At the genetic level, MM is characterized by both numerical and structural chromosomal alterations [1] 
alongside somatic gene mutations, as recently emerged from whole genome/exome sequencing analyses [3-6]. In fact, the general scenario arising from such studies indicated a considerable number of non-synonymous variants per patient, few recurrently mutated genes of probable pathogenetic significance, and a heterogeneous subclonal structure at the time of diagnosis [3-6]. Among the recurrently mutated genes with putative pathogenetic role, the most frequent were KRAS, NRAS, TP53, BRAF, TRAF3, FAM46C and DIS3 [3, 4, 6-8].

The DIS3 gene maps at 13q22.1 and encodes the catalytic subunit of the human RNA exosome complex, prominently participating to RNA processing and turnover [9]. DIS3 catalytic activity is governed by the endoribonucleolytic (PIN) and the 3'-5' exoribonucleolyitic (RNB) domains. It is indispensable for survival in vertebrates [10]. Somatic mutations and altered expression have been reported in several cancer types (COSMIC catalogue, Release v71, http://cancer. sanger.ac.uk/cancergenome/projects/cosmic/) $[11,12]$. Concerning MM, DIS3 was reported as mutated on average in $11 \%$ of patients $[4,6,7,13]$. Some DIS3 mutations identified in $\mathrm{MM}$ showed to interfere with its exonucleolytic activity, causing aberrant RNA metabolism and slower proliferation rate in HEK293derived human cell lines [10]. Furthermore, very recently we demonstrated that DIS3 facilitates the maturation of the tumor suppressor let-7 miRNAs by reducing in the cytoplasm the RNA stability of the pluripotency factor LIN28B, an inhibitor of let-7 processing. Through the reduction of mature let-7, DIS3 enhances the translation of let-7 targets such as MYC and RAS leading to enhanced tumorigenesis [14].

Here, we assessed by means of next-generation sequencing (NGS) the mutational status of DIS3 gene in a large and representative series of patients at different stages of plasma cell dyscrasia, and related it with other biological and clinical features; furthermore, we analyzed for the first time the gene expression pattern characterizing DIS3-mutated MM patients.

\section{RESULTS}

\section{Assessment of DIS3 mutations in PC dyscrasias}

To estimate the frequency of DIS3 mutations in different phases of plasma cell dyscrasia, 164 cases (including $130 \mathrm{MM}, 24 \mathrm{pPCL}$ and $10 \mathrm{sPCL}$ patients) and 20 human myeloma cell lines (HMCLs) were subjected to deep sequencing of the two functional domains of the gene, namely PIN and RNB domains.

The median depth of coverage was $245 x$ (range: 64-1160x). After exclusion of intronic, synonymous and germline variants, deep sequencing of DIS3 revealed 32 different coding somatic mutations globally targeting 27 genic positions (Table 1). The presence of each non-synonymous single nucleotide variant (SNV) or indel was validated in an independent PCR product, analyzed by conventional sequencing whenever the sensitivity of the Sanger method (i.e. about $10 \%$ in our experimental conditions) was consistent with the variant allele frequency (VAF), or by an additional ultra-deep pyrosequencing run (median depth of coverage $=1110 \mathrm{x}$ ) in case of variants at low allele frequency. At DNA level, 29 out of 32 variants $(90.6 \%)$ were SNVs, all resulting in missense mutations but one (73355968C > T, M1_V16del), which replaces one base within the first coding ATG, thus relocating the translation start site 16 codons downstream without altering the reading frame. The remaining three variants $(9.4 \%)$ were indels: two involved a single different nucleotide causing a frameshift, while the other was a $27 \mathrm{bp}$-deletion resulting in an in-frame deletion of nine amino acids (L48_A56del). Nonsense mutations were not observed. Two of the identified variants were exclusively carried by two HMCLs (see below).

As for primary patients, 21 out of 30 mutations (70\%) affected the RNB domain, while only eight variants $(8 / 30,26.7 \%)$ were located in the PIN domain. The remaining SNV mapped in a region just downstream of the RNB domain. Overall, the most affected residue was the arginine at position 780 (R780), replaced by a lysine or by a threonine in three and two cases, respectively (Figure 1). This is in line with what reported in previous MM sequencing studies, where this amino acid position was already indicated as the major DIS3 mutational hotspot $[4,6,7,13]$. The arginine at position 780 was found involved in RNA binding function [15]. Both R780K and R780T substitutions are recurrent in MM patients (of our and previously published datasets $[6,7,13])$, and the former was demonstrated to cause significant aberrations of DIS3 exonucleolytic activity and growth inhibition in yeast and human cellular models [10]. The second most affected residue in our dataset was the aspartic acid at position 488 (D488), involved in magnesium ion binding at the active site [15]. This residue was mutated in four cases, all carrying the D488N substitution, which also represents the globally most frequent variant in our series. Of note, D488 represents the second most affected residue in DIS3mutated MM patients when combining the results of the previous studies $[3,4,6,7,13]$. Interestingly, the adjacent conserved aspartic acid at position 487 is equally essential for magnesium binding, and when substituted with asparagine $(\mathrm{D} 487 \mathrm{~N})$ it was reported to completely abolish DIS3 exonuclease activity in yeast and human cellular models, causing molecular phenotypes comparable to the $D I S 3^{\mathrm{R} 780 \mathrm{~K}}$ mutant [10]. Notably, two missense variants involving D487 residue were detected in our dataset (D487V and D487H, each in one patient). These mutations were not previously reported in MM. Other recurrently mutated positions in our series were T93, H764, F775, R789 and R820, each targeted in two samples by the same 
Table 1: Summary of DIS3 non-synonymous/indel variants identified by NGS in the present dataset

\begin{tabular}{|c|c|c|c|c|c|c|c|c|}
\hline Variant* & AA change & $\begin{array}{c}\text { dbSNP } \\
\text { ID (v142)/ }\end{array}$ & $\begin{array}{c}\text { Previously } \\
\text { reported }\end{array}$ & & Mutated & samples ( & VAF) & \\
\hline $73346338 \mathrm{C}>\mathrm{T}$ & $\mathrm{D} 488 \mathrm{~N}$ & COSM158635 & {$[6,13]$} & $\begin{array}{l}\text { KMS27 } \\
(49.7 \%)\end{array}$ & $\begin{array}{l}\text { MM-343 } \\
(46.2 \%)\end{array}$ & $\begin{array}{c}\text { PCL-041 } \\
(45.7 \%)\end{array}$ & $\begin{array}{c}\text { MM- } \\
398 \\
(3.8 \%)\end{array}$ & $\begin{array}{c}\text { MM- } \\
042 \\
(4.6 \%)\end{array}$ \\
\hline $73336064 \mathrm{C}>\mathrm{T}$ & $\mathrm{R} 780 \mathrm{~K}$ & COSM329311 & {$[4,6,7,13]$} & $\begin{array}{c}\text { PCL-019 } \\
(47.7 \%)\end{array}$ & $\begin{array}{c}\text { PCL-042 } \\
(45.9 \%)\end{array}$ & $\begin{array}{c}\text { MM-281 } \\
(36.8 \%)\end{array}$ & & \\
\hline $73355093 \mathrm{~T}>\mathrm{C}$ & T93A & / & / & $\begin{array}{l}\text { PCL-021 } \\
(100 \%)\end{array}$ & $\begin{array}{l}\text { MM-213 } \\
(56.6 \%)\end{array}$ & & & \\
\hline $73335837 G>A$ & R820W & $\begin{array}{c}\text { rs372878316/ } \\
\text { COSM3469577 }\end{array}$ & l & $\begin{array}{l}\text { MM-445 } \\
(98.4 \%)\end{array}$ & $\begin{array}{c}\text { MM-207 } \\
(2.1 \%)\end{array}$ & & & \\
\hline $73335930 G>A$ & R789W & / & l & $\begin{array}{l}\text { MM-335 } \\
(94.2 \%)\end{array}$ & $\begin{array}{l}\text { PCL-011 } \\
(91.1 \%)\end{array}$ & & & \\
\hline $73336078 \mathrm{~A}>\mathrm{C}$ & F775L & l & {$[6]$} & $\begin{array}{c}\text { MM-036 } \\
(92.6 \%)\end{array}$ & $\begin{array}{c}\text { MM-414 } \\
(4.7 \%)\end{array}$ & & & \\
\hline $73336064 C>G$ & $\mathrm{R} 780 \mathrm{~T}$ & l & {$[6]$} & $\begin{array}{c}\text { PCL-035 } \\
(45 . \%)\end{array}$ & $\begin{array}{l}\text { MM-381 } \\
(16.4 \%)\end{array}$ & & & \\
\hline 73352393A $>C$ & V171G & l & $\begin{array}{l}\text { http://www. } \\
\text { keatslab.org }\end{array}$ & $\begin{array}{l}\text { KMS26 } \\
(100 \%)\end{array}$ & & & & \\
\hline $73336112 \mathrm{~T}>\mathrm{C}$ & H764R & l & / & $\begin{array}{c}\text { PCL-036 } \\
(97.6 \%)\end{array}$ & & & & \\
\hline $73337707 G>T$ & A670D & / & / & $\begin{array}{l}\text { PCL-001 } \\
(97 \%)\end{array}$ & & & & \\
\hline $73355010 \mathrm{G}>\mathrm{C}$ & F120L & l & / & $\begin{array}{c}\text { MM-123 } \\
(95.3 \%)\end{array}$ & & & & \\
\hline $73355048 G>A$ & $\mathrm{R} 108 \mathrm{C}$ & l & / & $\begin{array}{c}\text { MM-424 } \\
(94.3 \%)\end{array}$ & & & & \\
\hline $73355008 \mathrm{~T}>\mathrm{G}$ & Y121S & l & [19] & $\begin{array}{c}\text { OPM2 } \\
(91.8 \%)\end{array}$ & & & & \\
\hline $73355018 \mathrm{~T}>\mathrm{C}$ & $\mathrm{K} 118 \mathrm{E}$ & / & / & $\begin{array}{l}\text { PCL-015 } \\
(90.1 \%)\end{array}$ & & & & \\
\hline 73355804_73355830del & L48_A56del & l & / & $\begin{array}{c}\text { MM-340 } \\
(54.9 \%)\end{array}$ & & & & \\
\hline $73355968 \mathrm{C}>\mathrm{T}$ & M1_V16del & l & / & $\begin{array}{c}\text { MM-464 } \\
(51 \%)\end{array}$ & & & & \\
\hline $73336151 G>T$ & A751D & / & l & $\begin{array}{c}\text { MM-340 } \\
(45.7 \%)\end{array}$ & & & & \\
\hline $73346340 \mathrm{~T}>\mathrm{A}$ & D487V & / & l & $\begin{array}{l}\text { MM-317 } \\
(40.3 \%)\end{array}$ & & & & \\
\hline $73346341 C>G$ & D487H & / & l & $\begin{array}{c}\text { MM-372 } \\
(35.5 \%)\end{array}$ & & & & \\
\hline $73345240 G>A$ & S550F & l & / & $\begin{array}{c}\text { MM-386 } \\
(35.1 \%)\end{array}$ & & & & \\
\hline
\end{tabular}

(Continued) 


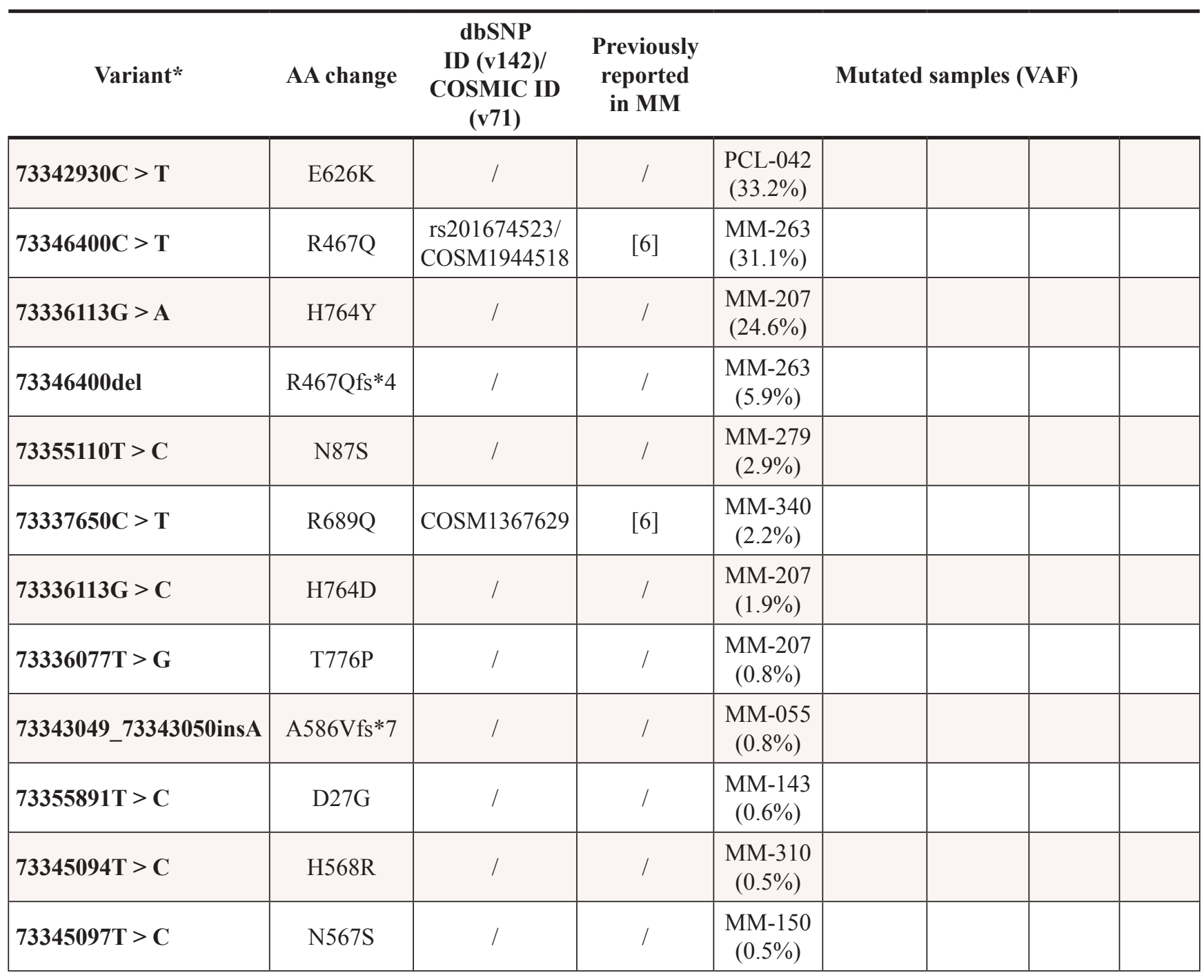

*Genomic positions based on hg19.

substitution, except for H764 (reported to be involved in RNA binding [15]) showing different amino acid changes. Notably, the majority of these recurrently altered residues represent novel DIS3 mutations in MM. In fact only the variant $\mathrm{F} 775 \mathrm{~L}$ has already been reported in another series [6]. The 17 remaining genic positions were found privately mutated in our cohort. Some of them have been already reported as altered in MM (R108, R467, S550) [6] or in other cancer types (R820, COSM3469577); among the mutated residues never described before, A751 is involved in RNA binding [15]. Similar to other studies [3, 4, 6, 7], we did not find any mutation involving the residue E665, proposed as mutational hotspot by Weißbach and colleagues based on their sequencing results [13].

Notably, four patients carried multiple DIS3 mutations; only in two of these cases (MM-340 and PCL-042) two variants were both at high allele frequency, while in the remaining samples only one of the mutations was above the detection limit of Sanger method, accompanied by other variants at low allele frequency.
In particular, PCL-042 harbored the variants R780K and E626K, both in the RNB domain, in $46 \%$ and $33 \%$ of reads, respectively, and MM-340 carried a nine amino acid in-frame deletion in the PIN domain (L48_A56del) and a missense substitution in the RNB domain (A751D) in 55\% and $46 \%$ of reads, respectively.

Beyond the aforementioned R780 and D487 residues, whose importance for the catalytic activity of the protein has been functionally proven, a potential detrimental effect on DIS3 nucleolytic activity can be suggested also for many of the other mutations we identified here, on the basis of their recurrence (as described above), and/or the high conservation of affected amino acid residues, and/or the functional predictions returned by different algorithms. In fact, from the alignment of human DIS3 protein sequence to mouse and yeast paralogs and to E.coli RNases II and $\mathrm{R}$ sequences, we found that 13 of the 24 genic positions targeted by mutations resulting in amino acid substitutions or in-frame deletions in our primary patients encoded 


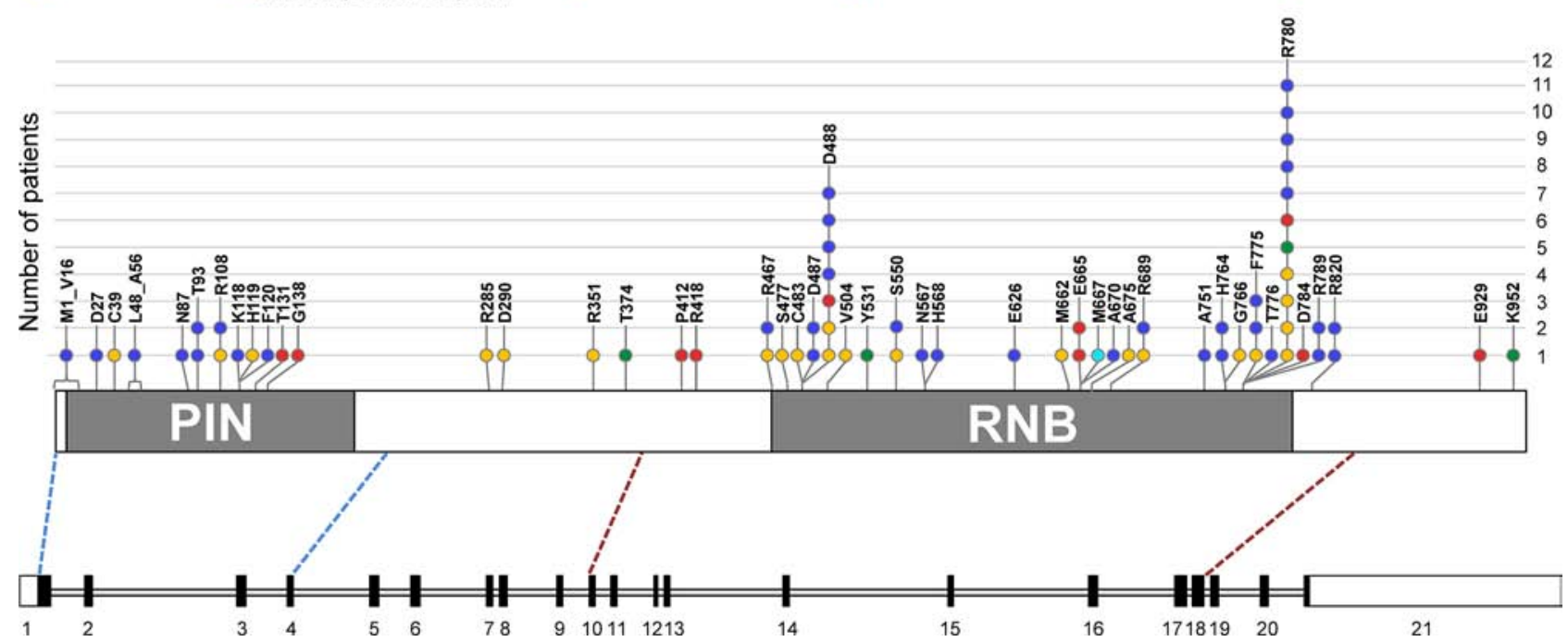

Figure 1: Frequency of SNVs and in-frame deletions in DIS3 coding region in MM, including information from literature. In the lower part of the figure, a schematic diagram of the DIS3 gene is reported, based on exon-intron organization of the longer transcript variant, NM_014953.4. Exons are numbered under the boxes, filled with black in the coding region. Exons sequenced in this study (i.e., exons 1-4 and exons 10-18) encompass respectively the entire PIN (cd09862, amino acids 9-197) and RNB domain (pfam00773, amino acids 467-791), plus portions of the flanking regions, as indicated by the blue and red dashed lines connecting the diagram of the gene to the scheme of the DIS3 protein, in the upper part of the figure. Here, somatic mutations identified in the present series and reported in the main MM datasets $[3,4,6,7,13]$ are depicted.

amino acid residues conserved in all the analyzed species (Supplementary Figure S1). Furthermore, 21 out of the 26 missense mutations were suggested to be functionally relevant according to five different prediction tools (Supplementary Table S1).

Regarding DIS3 mutations found in the cell lines, three out of the 20 analyzed HMCLs were mutated for this gene. In particular, KMS27 carried the aforementioned D488N hotspot mutation, while OPM2 and KMS26 cells harbored the variants Y121S and V171G, respectively. Both these substitutions were located in the PIN domain and were absent in primary tumors of both our and other series; only the $\mathrm{V} 171 \mathrm{G}$ involved a highly conserved residue and was suggested to be functionally relevant by all the bioinformatics predictors. Our data concerning HMCLs are concordant with those reported in the MM Cell Line Characterization Project (available at http:// www.keatslab.org/data-repository).

\section{DIS3 mutations in different phases and molecular subtypes of PC dyscrasia}

DIS3 mutations globally affected 33 patients (33/164, 20.1\%): specifically, $24 \mathrm{MMs}$ at diagnosis (24/130, $18.5 \%$ ), six primary PCLs $(6 / 24,25 \%)$, and three secondary PCLs (3/10, 30\%). The most relevant molecular features of the 33 mutated cases are reported in Supplementary Table S2.

We next asked whether there were any relationships with cytogenetic lesions. We observed a positive association between DIS3 mutations and
IGH@ translocations $(P$ value $=0.0046)$, particularly with the $\mathrm{t}(11 ; 14)$ ( $P$ value $=0.0198)$, and a lower occurrence of hyperdiploidy among DIS3- mutated cases $(P$ value $=0.025)$. Conversely, we were unable to find any association with $17 \mathrm{p}$ deletion, 1q gain or $1 \mathrm{p}$ loss (Table 2). Although not significantly associated with 13q14 deletion, DIS3 mutations occurred in several cases carrying this chromosomal alteration. Specifically, 19 of the 33 (57.6\%) DIS3-mutated patients harbored del(13q14). For eight of them, genome-wide DNA profiling data were also available $[16,17]$, indicating that in all but one case (PCL-019) the chromosomal deletion included the DIS3 locus. Interestingly, VAFs around $100 \%$ were exclusively observed in a fraction of patients (10/19) with $13 q$ deletion (and in all likelihood with heterozygous deletion of the DIS3 locus). In the other cases, i.e. the remaining del(13q14) samples (9/19) and all the 14 patients disomic for chromosome 13, the VAFs we found were indicative of mutations occurring in hemizygosis in tumor subclones or carried in heterozygosis either by all or by a fraction of myelomatous cells (Supplementary Figure S2).

Variants detected at very low allele frequencies included both missense and frameshift mutations; some of these low-level variants (i.e. D488N, F775L, R820W) were also carried at higher VAFs in other patients. Interestingly, substitutions at residues R780, D487 and D488 (demonstrated or suggested to be particularly detrimental for DIS3 protein function) were never observed at VAFs higher than 50\%, neither in the present nor in previously published datasets $[6,7,13]$. 
Table 2: Clinical and molecular characteristics of the $164 \mathrm{MM} / \mathrm{PCL}$ patients analyzed for DIS3 mutations

\begin{tabular}{|c|c|c|c|c|c|c|c|}
\hline \multirow[t]{2}{*}{ Characteristic } & \multicolumn{2}{|c|}{ All patients $(n=164)$} & \multicolumn{2}{|c|}{$D I S 3$ wild type $(n=131)$} & \multicolumn{2}{|c|}{ DIS3-mutated $(n=33)$} & \multirow[t]{2}{*}{$P$ value ${ }^{a}$} \\
\hline & $\mathbf{N}$ & $\%$ & $n$ & $\%$ & $n$ & $\%$ & \\
\hline MM & 130 & 79 & 106 & 81 & 24 & 73 & \multirow{3}{*}{ n.s. } \\
\hline pPCL & 24 & 15 & 18 & 14 & 6 & 18 & \\
\hline sPCL & 10 & 6 & 7 & 5 & 3 & 9 & \\
\hline $\operatorname{del}(13 q)$ & 77 & 47 & 58 & 45 & 19 & 58 & \multirow{2}{*}{ n.s. } \\
\hline $\begin{array}{l}\text { chr } 13 \text { disomic } \\
\text { patients }\end{array}$ & 86 & 53 & 72 & 55 & 14 & 42 & \\
\hline $\operatorname{del}(17 p)$ & 17 & 10.5 & 15 & 12 & 2 & 6 & \multirow{2}{*}{ n.s. } \\
\hline $\begin{array}{l}17 \text { p disomic } \\
\text { patients }\end{array}$ & 145 & 89.5 & 114 & 88 & 31 & 94 & \\
\hline 1q gain & 66 & 43 & 49 & 39 & 17 & 57 & \multirow{2}{*}{ n.s. } \\
\hline $\begin{array}{l}\text { 1q disomic } \\
\text { patients }\end{array}$ & 89 & 57 & 76 & 61 & 13 & 43 & \\
\hline $1 \mathrm{p}$ loss & 18 & 13 & 16 & 14 & 2 & 7 & \multirow{2}{*}{ n.s. } \\
\hline $\begin{array}{l}1 \mathrm{p} \text { disomic } \\
\text { patients }\end{array}$ & 123 & 87 & 97 & 86 & 26 & 93 & \\
\hline $\begin{array}{l}I G H \\
\text { translocation }\end{array}$ & 81 & 50 & 58 & 44 & 23 & 74 & \multirow[t]{2}{*}{0.0046} \\
\hline $\begin{array}{l}\text { no } I G H \\
\text { translocation }\end{array}$ & 81 & 50 & 73 & 56 & 8 & 26 & \\
\hline hyperdiploid & 49 & 33 & 45 & 37 & 4 & 14 & \multirow{2}{*}{0.0250} \\
\hline $\begin{array}{l}\text { non- } \\
\text { hyperdiploid }\end{array}$ & 101 & 67 & 77 & 63 & 24 & 86 & \\
\hline
\end{tabular}

aSignificance was assessed by Freeman-Halton extension of Fisher's exact test for disease type, and by Fisher's exact test for all other variables. n.s.: not significant.

\section{Longitudinal analysis of DIS3 mutations}

To gain further insight into the state of DIS3 mutations longitudinally, we analyzed 19 patients for whom BM specimens were collected at two different timepoints: in particular, fourteen MM and two pPCL cases collected both at onset and at relapse; two MM patients at onset and at leukemic transformation, and one patient at early and relapsed leukemic phase of MM (Figure 2, Supplementary Table S3). Fifteen out of the 19 cases displayed a wild type DIS3 status at both timepoints, while one patient (MM-281) carrying the $\mathrm{R} 780 \mathrm{~K}$ variant showed a quite constant VAF during disease course (36.8\% mutated reads at diagnosis, and $47.56 \%$ at leukemic transformation). MM-004, wild type at onset, acquired a missense mutation affecting a fully conserved amino acid residue downstream the RNB domain and suggested to be of functional relevance (A827P); this SNV was detected in $97.1 \%$ of the sequencing reads at relapse. This patient showed two copies of chromosome 13 both at onset and at relapse, and also the other cytogenetic lesions remained stable during disease progression. A considerable increase in mutation burden was observed in MM-263, with R467Q mutation occurring in $31.1 \%$ of reads at onset and $81.9 \%$ at relapse (this case also carried a frameshift mutation at the same position with VAF $=5.93 \%$ at diagnosis, which disappeared at relapse). Finally, MM-340 carried three variants at onset (L48_A56del, A751D and R689Q at VAF of $54.86 \%, 45.71 \%$ and $2.20 \%$, respectively), two of which (L48_A56del and A751D) remained stable while the third (R689Q) was no longer detectable at relapse. The disappearance of mutations at high allele frequency at onset was never observed in our cohort.

\section{Expression of DIS3 variants}

To verify whether the DIS3 mutations here detected were expressed at transcriptional level, we 
performed the deep sequencing of cDNA samples of the DIS3-mutated cases for whom biological material was available. In particular, $20 \mathrm{DIS} 3$ variants were evaluated at transcriptional level (Figure 3A). Notably, all the variants detected at VAFs higher than $6 \%$ on genomic DNA were also identified at RNA level, while none of the four tested low-frequency genomic mutations (VAFs ranging from $1.95 \%$ to $5.96 \%$ ) was detected on cDNA. Overall, as regards expressed variants, mutant allele frequencies detected at gene and transcript level showed a good correlation (Figure 3B). Notably, the aforementioned increase in mutation burden from diagnosis to relapse observed in MM-263 for the variant R467Q was accompanied by a doubling of the amount of expressed mutant allele.

\section{Transcriptomic profile of DIS3-mutated patients}

To identify transcriptional gene profiles related to DIS3 mutations, we investigated by microarray technology a large fraction of MM samples of the present series, including both DIS3-mutated and wild type patients. Assuming that alterations present in a very limited number of malignant plasma cells might not appreciably affect gene expression, we chose to set at $20 \%$ the lower VAF cut-off to perform supervised analyses. We thus compared 89 wild type and 13 mutated patients for DIS3. This analysis revealed that 119 genes $(q$-value $<0.1)$ (Supplementary Table S4) were differentially expressed between the two groups. Notably, all transcripts were up-regulated in mutated cases (28 of these genes emerged at $q$-value $=0-$ Figure 4$)$. Interestingly, 74 were proteincoding genes, while one gene was of unknown type and 44 were non-coding RNAs/pseudogenes. In particular, this latter class of modulated transcripts included nine antisense RNAs (ARHGAP5-AS1, C21orf119, DLGAP1-AS1, HEXA-AS1, ILF3-AS1, LOC645212, MATN1-AS1, TAPT1-AS1, and ZNRD1-AS1), seven pseudogenes (CATSPER2P1, FLJ37201, GNRHR2, GVINP1, HTATSF1P2, NUDT9P1, and PRORSD1P), six long intergenic non-protein-coding RNAs (FLJ30403, TP53TG1, LINC00167, LINC00173, LINC00528, and LOC100129726), five small nuclear RNAs (RNU11, $R N U 12, R N U 4 A T A C, R N U 5 D-1$, and $R N U 5 F-1)$, two microRNAs (MIR23A and MIR320A), one microRNAhost gene (MIRLET7BHG), and a group mainly consisting of uncharacterized loci and open reading frames. We then explored whether these transcripts belonged to specific functional subgroups. Remarkably, a significant fraction of them was involved, like DIS3 itself, in single-stranded RNA binding function (namely, PAT2L, DHX58, DDX60 and KHDRBS2; $P$ value $=1.01 \mathrm{E}-04)($ Table 3$)$. Beyond this specific molecular function, several other up-regulated genes in DIS3-mutated patients are implicated in RNA physiology: in particular, $P A N 2, P O P 1, R B M 45$ and the aforementioned PATL2 and KHDRBS2 genes are involved in RNA metabolism; $A P O B E C 3 F$ and $A P O B E C 4$ encode RNA editing enzymes; whereas RNU11, RNU12 and RNU4ATAC are spliceosome subunits. Notably, $D H X 58$, $D D X 60$ and $A P O B E C 3 F$ genes, along with IFIT1, IFIT3, $I F I 6$, and $O A S L$, are part of interferon-mediated anti-viral response.

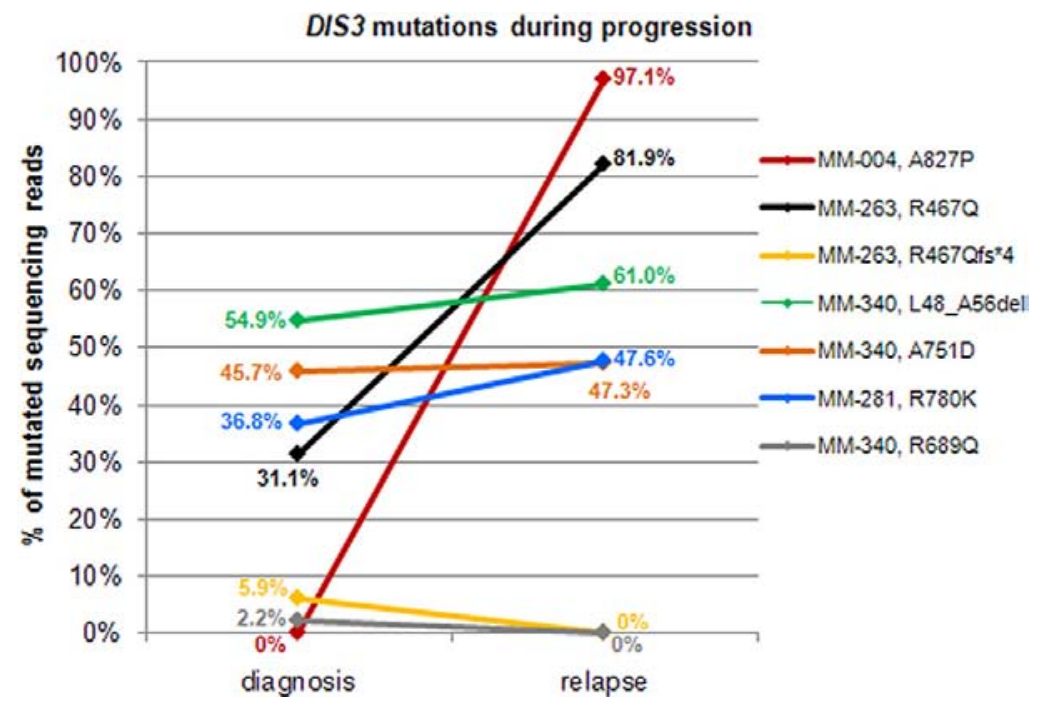

Figure 2: Changes of DIS3 mutational burden during disease progression. For patients found mutated at diagnosis and/or relapse, allele frequencies of variants reported in the legend are plotted at both timepoints. 

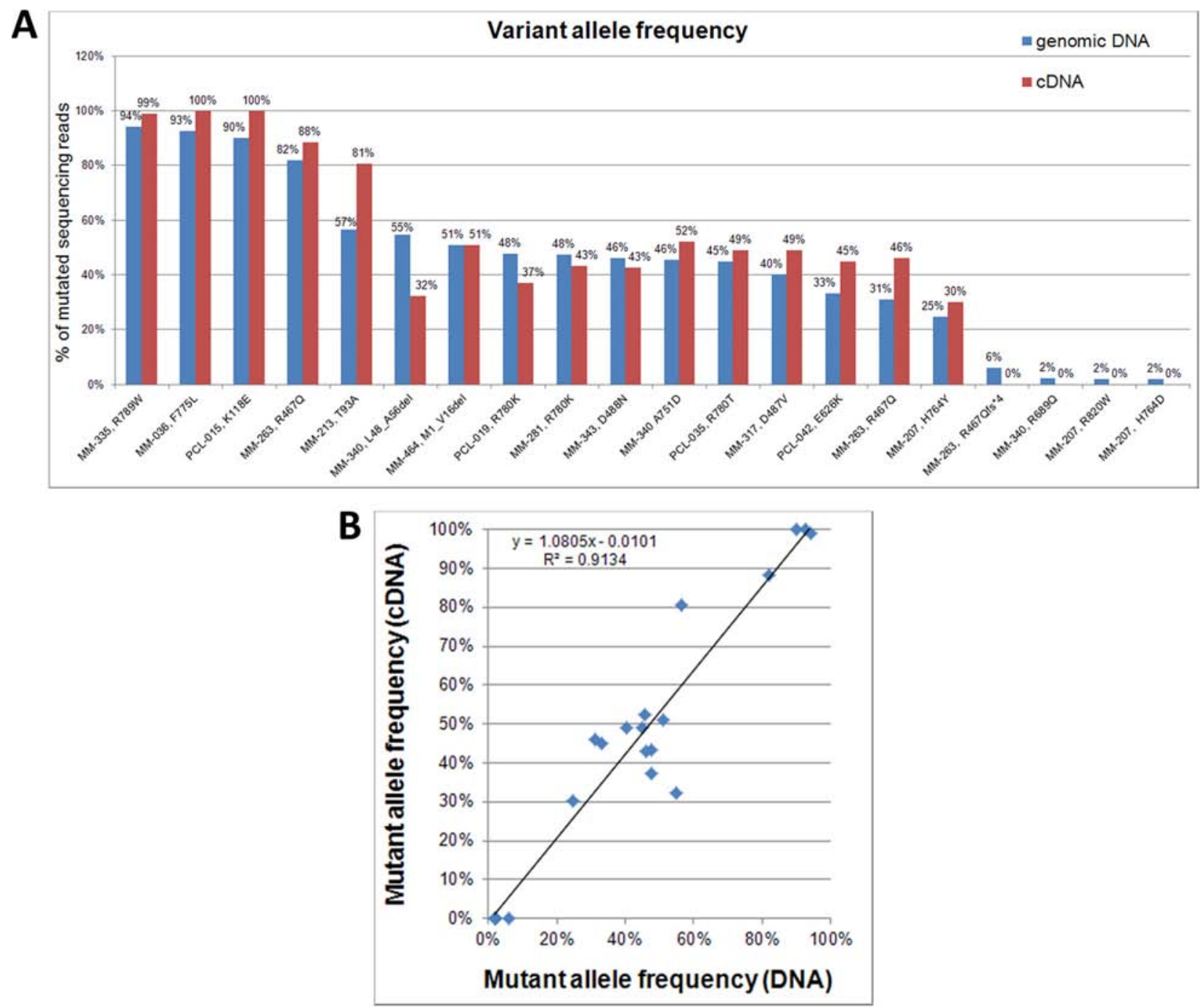

Figure 3: DIS3 mutations detected on genomic DNA and cDNA. A. Percentages of variant DIS3 sequencing reads identified by NGS analyses of genomic DNA and retrotranscribed total RNA. B. Correlation between VAFs detected on genomic DNA and cDNA.

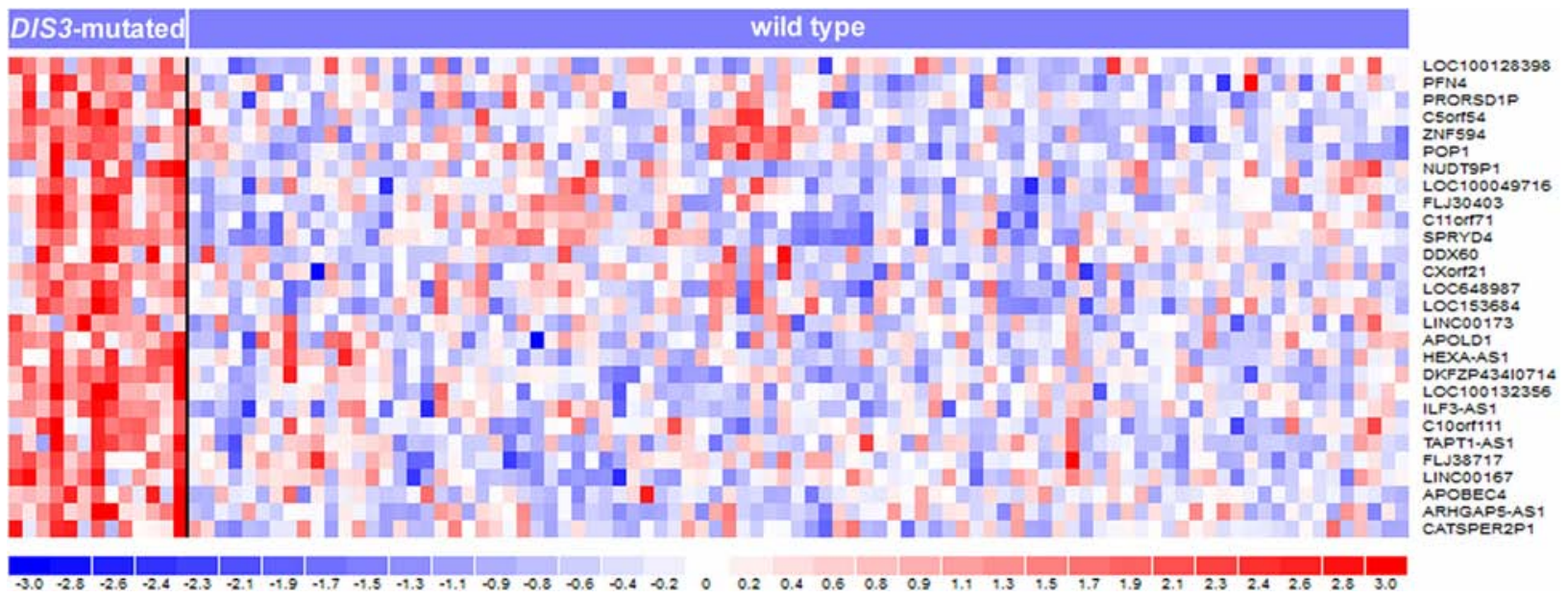

Figure 4: Heatmap of the 28 differentially expressed genes identified at $q$-value $=0$ by SAM two-class analysis of 102 MM patients stratified based on the presence of $D I S 3$ mutations. In the heatmap, the color-scale bar represents the relative gene expression changes normalized by the standard deviation, and the color changes in each row represent gene expression level relative to the mean across the samples. 
Table 3: Selected significantly enriched gene ontology terms in the DIS3 mutation-associated gene signature ( $q$-value $<0.05$, Benjamini Hochberg correction)

\begin{tabular}{|c|c|c|c|}
\hline Category & Name & $q$-value & Genes \\
\hline GO: Molecular Function & single-stranded RNA binding & 2.01E-02 & PATL2, DHX58, DDX60, KHDRBS2 \\
\hline GO: Biological Process & $\begin{array}{l}\text { positive regulation of MDA-5 } \\
\text { signaling pathway }\end{array}$ & 3.32E-02 & $D H X 58, D D X 60$ \\
\hline GO: Biological Process & $\begin{array}{l}\text { regulation of MDA-5 } \\
\text { signaling pathway }\end{array}$ & 3.32E-02 & $D H X 58, D D X 60$ \\
\hline GO: Biological Process & $\begin{array}{l}\text { positive regulation of RIG-I } \\
\text { signaling pathway }\end{array}$ & 3.32E-02 & DHX58, DDX60 \\
\hline GO: Biological Process & defense response to virus & $3.32 \mathrm{E}-02$ & $\begin{array}{c}\text { DHX58, DDX60, IFIT1, IFIT3, APOBEC } 3 F, \\
\text { OASL }\end{array}$ \\
\hline GO: Biological Process & $\begin{array}{l}\text { cellular response to type I } \\
\text { interferon }\end{array}$ & 3.32E-02 & IFIT1, IFIT3, OASL, IFI6 \\
\hline GO: Biological Process & $\begin{array}{c}\text { type I interferon signaling } \\
\text { pathway }\end{array}$ & 3.32E-02 & IFIT1, IFIT3, OASL, IFI6 \\
\hline GO: Biological Process & MDA-5 signaling pathway & 3.32E-02 & DHX58, DDX60 \\
\hline GO: Biological Process & response to type I interferon & 3.32E-02 & IFIT1, IFIT3, OASL, IFI6 \\
\hline GO: Biological Process & $\begin{array}{l}\text { regulation of defense } \\
\text { response to virus }\end{array}$ & $3.76 \mathrm{E}-02$ & DHX58, DDX60, IFIT1, APOBEC $3 F$ \\
\hline Pathway (REACTOME) & $\begin{array}{l}\text { Interferon alpha/beta } \\
\text { signaling }\end{array}$ & $1.01 \mathrm{E}-04$ & IFIT1, IFIT3, OASL, IFI6 \\
\hline
\end{tabular}

\section{DISCUSSION}

DIS3 belongs to the human exosome complex and is endowed with both exo- and endonucleolytic activities. DIS3 regulates the processing and abundance of all RNA species [18]. Recently, DIS3 gene has emerged as recurrently mutated in MM patients from initial wholegenome (WGS) and exome (WES) sequencing studies $[4,6,7,13,19]$. To the best of our knowledge, this is the first study assessing the DIS3 mutational status by means of NGS technology in a large cohort of patients including different molecular subtypes and phases (also leukemic stages) of PC dyscrasias and comprehensively evaluating it in the context of other molecular features, and determining the transcriptional profiles and putative altered pathways in mutated MM patients.

DIS3 mutations were globally identified in $20.1 \%$ of patients: specifically, mutation prevalence was $18.5 \%$ in newly diagnosed MM patients, $25 \%$ in pPCL at onset, and $30 \%$ in sPCL. Interestingly, DIS3 mutation frequency seemed to be slightly higher in extramedullary phases (although this difference did not reach statistical significance, likely due to the relatively small size of pPCL and SPCL series). Notably, a WES study in a fraction of the pPCL patients of the present series identified DIS3 as one of the 14 statistically significant recurrently affected genes with potential driver role in the disease [20]. As regards the prevalence of $D I S 3$ mutations identified here in representative $\mathrm{MM}$ cases at onset (18.5\%), it was higher than the one reported for unselected patients' cohorts in other studies $[4,6,13]$. Most likely, this could be due to the higher depth of coverage of our sequencing analysis, especially compared to that usually obtained in WGS and WES experiments. Compared to the lower frequency (11\%) reported by Weißbach et al. [13], who used our same targeted resequencing approach, this difference could be due to the exclusion in that study of the variants with VAF lower than $8 \%$. We obtained similar results as of Walker et al. (18\%) [7], who exclusively analyzed IGHtranslocated patients. Indeed, also in the present series, DIS3 mutations were confirmed preferentially associated with $I G H$ translocations and nonhyperdiploid status.

Of the two functional domains investigated in this study, the RNB domain was slightly more densely affected by mutations. The mutations of the RNB domain initially identified in MM by Chapman et al. [4] interfere with DIS3 ribonucleolytic activity [10]. This functional effect could be envisaged also for many of the mutations identified by us, based on the bioinformatics predictions and the pattern of differential expression between DIS3mutated and wild type MM cases emerged from the gene expression profiling of our cohort. DIS3-mutated MM 
samples, in fact, displayed the up-regulation of several transcripts, including genes involved in RNA interactions and many non-coding transcripts. The finding that all the involved transcripts were over-expressed in DIS3-mutated patients reflects what reported by several studies analyzing the transcriptional output resulting from the mutation or depletion of exosome subunits in other species, and is consistent with the RNA degradative activities ascribed to the exosome [21-23]. Also the nature of these transcripts, in particular for non-protein-coding RNAs, could be compatible with the accumulation of physiological exosome substrates due to the loss of function of DIS3. In fact, we could notice some similarities between the transcriptional profile characterizing our DIS3-mutated MM patients and the genome-wide atlas of Arabidopsis thaliana exosome targets presented by Chekanova et al., including hundreds of non-coding RNAs, most of which have not been previously described [21]. Among those targets, Chekanova et al. reported upstream non-coding transcripts (UNTs), rRNA precursors, snoRNAs, snRNAs, pseudogenes, pri-miRNAs, and transcripts having neither protein-coding potential nor predicted function [21]. Interestingly, as reported by Tomecki et al., Northern blot analyses in HEK293 cells expressing MM-associated DIS3 mutations (such as $\mathrm{R} 780 \mathrm{~K}$ ) revealed the accumulation of the majority of analyzed exosome substrates, including 5.8S processing intermediates, tRNAs, RNA polymerase III transcripts and PROMPTs (i.e. PROMoter uPstream Transcripts), these latters generated 0.5 to $2.5 \mathrm{~kb}$ upstream of active transcription start sites and rapidly turned over by the RNA exosome [10]. The accumulation of similar nuclear non-coding RNAs (such as cryptic unstable transcripts, CUTs) when MM DIS3 mutations were expressed in yeast led the same Authors to speculate that the level of 'transcriptional noise' is probably much higher in the nucleus of the cells producing DIS3-mutated variants. Concerning the up-regulation of genes involved in interferon-mediated anti-viral response observed in the DIS3-mutated MM patients of our cohort, a potential parallel might be suggested between the induction of antiviral transcriptional response found associated with DIS3 mutations in our study and the recently reported evidence that SKIV2L, the helicase component of the RNA exosome, is involved in the degradation of endogenous RNAs that might inappropriately trigger the RLRs (RIGI-like receptors), which are the cytosolic sensors of viral RNAs that initiate host defense, including secretion of type I interferons. Thus, SKIV2L prevents aberrant innate immune responses; remarkably, humans with deficiency in SKIV2L have a type I interferon signature in their peripheral blood [24].

The gene expression patterns observed in our study suggested that identified mutations here are actually expressed, as directly assessed by cDNA sequencing of DIS3-mutated patients showing comparable mutation burdens at gene and transcript levels. Overall, these data demonstrate the ultimate significance of the DIS3 gene mutations, differently from what has been suggested for the majority of the genetic changes detected by genomewide approaches in MM, which are found in genes that have low or no detectable biological expression [25]. In this regard, it is also worth noting that substitutions at residues R780, D487 and D488 (demonstrated or supposed to be particularly detrimental for protein function) were never observed at genomic VAFs above 50\%, neither in the present nor in previously published datasets $[6,7,13]$, suggesting that the exclusive presence of such DIS3 mutant forms in the absence of a DIS3 wild type protein might have deleterious cellular effects. In general, the lack of a DIS3 wild type allele in mutated cases was uniquely observed in some 13q14-deleted MM patients carrying other types of variants.

Furthermore, looking at DIS3 mutations in longitudinally analyzed patients (i.e. sampled at diagnosis and relapse), we observed a similar scenario to that described by Bolli et al. [3] for other known driver myeloma genes, i.e. the occurrence of clonal variants at both timepoints or the acquisition/clonal expansion of variants at the later timepoint, consistently with an expected positive selection for the subclones harboring them. Of note, the disappearance at relapse of variants found at diagnosis at low allele frequency was only observed in patients carrying additional stable/clonally expanding mutations. The limited sample size and the different treatment regimens to which the patients were subjected do not allow making assumptions on the possible impact of specific therapies on the chances of survival of DIS3-mutated subclones.

The clinical implications of DIS3 mutations in $\mathrm{MM}$ remain to be elucidated. Recently, Weißbach et al. [13] reported that the occurrence of DIS3 mutations in minor subclones was significantly associated with a weaker chemotherapy response as compared to the DIS3 mutations in major subclones, while globally there was only a slight trend towards shorter median overall survival for DIS3-mutated MM patients as compared to DIS3 wild type ones. In our series, we had the possibility to evaluate the prognostic impact of DIS3 mutations in the group of pPCLs included in a prospective multicenter clinical trial [26] for whom a 2.8-month follow-up was available, observing no significant association with survival or response rate (data not shown).

In conclusion, our data confirm and extend the evidence that DIS3 mutations are recurrent in MM, suggesting an even greater involvement of DIS3 alterations in more advanced stages of PC dyscrasias, and highlighting some instances of increase of DIS3 mutation burden during disease progression. Although its role in the pathogenesis of the disease remains to be further elucidated, DIS3 is considered a potential tumor 
suppressor in MM. This suggestion is based on the loss of enzymatic activity caused by the MM-associated DIS3 mutations that have been functionally characterized [10]; the loss of heterozigosity/disruption (as also elucidated in our study) often involving DIS3 due to the combination of gene mutations and chromosome 13 deletion; and the increased translation of critical oncogenes observed as one of the biological outcomes of DIS3 inactivation [14]. Importantly, the transcriptional phenotype observed here in DIS3-mutated MM patients could be consistent with an impaired function of the RNA exosome complex, thus further supporting the pathological role of DIS3 alterations in the disease.

\section{MATERIALS AND METHODS}

\section{Patients and cell lines}

After the patients had given their informed consent in accordance with institutional guidelines (clearance from Ethic Committee, Fondazione Ospedale Maggiore di Milano, Italy), pathological BM specimens were obtained during standard diagnostic procedures from 130 newly diagnosed MM patients, 8 pPCL cases at onset, and 10 sPCLs, admitted from July 2001 to April 2014. Additional 16 pPCL patients were included in a multicenter clinical trial (RV-PCL-PI-350, EudraCT N²008-00324628) [26]. Seventy-seven patients were males; median age was 66 years (range: 42-85). The diagnosis of MM and PCL was made according to the previously described criteria $[2,27]$. Ninety-nine patients had an immunoglobulin (Ig) $\mathrm{G}$ protein monoclonal component; $34 \mathrm{IgA}$; one $\mathrm{IgG} / \mathrm{IgA}$; and one IgM protein; 99 cases had the light chain $\kappa ; 62 \lambda$; and two $\lambda+\kappa$. Twenty-five MM cases were in stage IA, 57 in IIA/B and 48 in IIIA/B, according to Durie and Salmon criteria [28]. Many of the patients included in this study have been described in our previous reports [29, 30].

HMCLs investigated in this study were the following: NCI-H929, OPM2, JJN3, KMS-12, KMS-28, KMS-34, KMS-18, KMS-11, KMS-26, AMO1, RPMI 8226, DELTA-47, SK-MM-1, UTMC-2, MM.1S, U266, CMA-03 and CMA-03/06, LP-1, KMS-27. With the exception of DELTA-47, UTMC-2 and MM.1S, all the others have been previously described and reported by us $[31,32]$.

\section{Sample preparation and molecular analyses}

The BM specimens were collected from patients at the time of diagnosis; 19 cases were re-sampled at relapse/MM leukemic transformation (median time interval between the two samplings: 30 months). PCs were purified ( $\geq 90 \%$ in all cases) using CD138 immunomagnetic microbeads as previously described [33, 34]. All cases were characterized by fluorescence in situ hybridization (FISH) for the main genomic aberrations (namely, IGH translocations, hyperdiploidy, del(13q), del(17p), del(1p) and 1q gain) [16] (Supplementary Table S5), and by NGS for the mutational status of $B R A F, N R A S$ and $K R A S$ genes [8].

\section{Mutation analyses}

NGS of DIS3 exons 1-4 (PIN domain) and exons 10 18 (RNB domain) (RefSeq NM_014953.4, representing the longer transcript encoding the longest protein isoform) was performed on genomic DNA using the Genome Sequencer Junior instrument (Roche-454 Life Sciences, Penzberg, Germany), as previously described [35]. Further details on primer sequences and sequencing protocol are available in the Supplementary Materials and Methods and Supplementary Table S6 and S7. The obtained sequencing reads were mapped to the DIS3 human reference sequence (RefSeq NC 000013.10) and analyzed by the Amplicon Variant Analyzer (AVA) software version 3.0 (454 Life Sciences) to establish the variant allele frequency.

The presence of each obtained non-synonymous variant was verified in an independent PCR product by conventional sequencing whenever the sensitivity of the Sanger method was consistent with the VAF. NGS analysis was repeated in case of mutations detected in less than $10 \%$ of sequencing reads. To exclude germline variants, we sequenced the matched normal DNA, when available, or consulted the NCBI Human dbSNP Database (Build 142, http://www.ncbi.nlm.nih.gov/snp). The occurrence of DIS3 variants was also verified at transcriptional level (see Supplementary Materials and Methods).

Additional information about the functional relevance of the detected SNVs was obtained using five bioinformatics tools which calculate possible impact of amino acid substitutions on the structure and function of human proteins using straightforward physical and evolutionary comparative considerations. The functional predictors used were Polyphen2 (http://genetics.bwh.harvard.edu/pph2/), Mutation Taster (http://www.mutationtaster.org/), Mutation Assessor (http:// mutationassessor.org/), SIFT (http://sift.jcvi.org/) and LRT (http://www.genetics.wustl.edu/jflab/lrt_query. html).

\section{Gene expression profiling}

For gene expression profiling (GEP), samples were profiled on the GeneChip Human Gene 1.0 ST array (Affymetrix, Santa Clara, CA, USA), as previously described [30]. Supervised analyses were performed using Significant Analysis of Microarrays software (SAM version 4.00; Excel front-end publicly available at http://www-stat.stanford.edu/tibs/SAM/index. html) [36]. The functional annotation analysis of selected gene lists was performed by means of DAVID 6.7 tool (http://david. abcc.ncifcrf.gov/) and the ToppFun option of ToppGene Suite (https://toppgene.cchmc.org/), using the default 
parameters. Details on GEP data generation and analysis are included in the Supplementary Materials and Methods. The GEP data have been deposited in the NCBI Gene Expression Omnibus database (GEO; http//www.ncbi.nlm .nih.gov/geo; accession No. GSE66293).

\section{Statistical analyses}

All contingency analyses were performed by twosided Fisher's exact test $(P$ value $<0.05)$.

\section{ACKNOWLEDGMENTS}

This work was financially supported by grants from: the AIRC Investigator Grants no.10136 (to A.N.), and no.9256 and no.13103 (to G.T.), and the AIRC Special Program Molecular Clinical Oncology, 5 per mille no. 9980 to A.N. and P.T., and no. 9965 to G.T.; and the Ministero Italiano dell'Istruzione, Università e Ricerca (MIUR) grant 2009PKMYA2 (to A. N.). P. M. is supported by a Research fund from Celgene. K. T. was supported by the Italian Health Minister, Finalized Research for Young Researchers, CUP Project E66110000230001.

\section{CONFLICTS OF INTEREST}

The authors declare that they have no conflict of interest.

\section{REFERENCES}

1. Morgan GJ, Walker BA, Davies FE. The genetic architecture of multiple myeloma. Nat Rev Cancer. 2012; 12:335-348.

2. Albarracin F, Fonseca R. Plasma cell leukemia. Blood Rev. 2011; 25:107-112.

3. Bolli N, Avet-Loiseau H, Wedge DC, Van Loo P, Alexandrov LB, Martincorena I, Dawson KJ, Iorio F, Nik-Zainal S, Bignell GR, Hinton JW, Li Y, Tubio JM, et al. Heterogeneity of genomic evolution and mutational profiles in multiple myeloma. Nat Commun. 2014; 5:2997.

4. Chapman MA, Lawrence MS, Keats JJ, Cibulskis K, Sougnez C, Schinzel AC, Harview CL, Brunet JP, Ahmann GJ, Adli M, Anderson KC, Ardlie KG, Auclair D, et al. Initial genome sequencing and analysis of multiple myeloma. Nature. 2011; 471:467-472.

5. Keats JJ, Chesi M, Egan JB, Garbitt VM, Palmer SE, Braggio E, Van Wier S, Blackburn PR, Baker AS, Dispenzieri A, Kumar S, Rajkumar SV, Carpten JD, et al. Clonal competition with alternating dominance in multiple myeloma. Blood. 2012; 120:1067-1076.

6. Lohr JG, Stojanov P, Carter SL, Cruz-Gordillo P, Lawrence MS, Auclair D, Sougnez C, Knoechel B, Gould J, Saksena G, Cibulskis K, McKenna A, Chapman MA, et al. Widespread genetic heterogeneity in multiple myeloma: implications for targeted therapy. Cancer Cell. 2014; 25:91-101.

7. Walker BA, Wardell CP, Melchor L, Hulkki S, Potter NE, Johnson DC, Fenwick K, Kozarewa I, Gonzalez D, Lord CJ, Ashworth A, Davies FE, Morgan GJ. Intraclonal heterogeneity and distinct molecular mechanisms characterize the development of $\mathrm{t}(4 ; 14)$ and $\mathrm{t}(11 ; 14)$ myeloma. Blood. 2012; 120:1077-1086.

8. Lionetti M, Barbieri M, Todoerti K, Agnelli L, Marzorati S, Fabris S, Ciceri G, Galletti S, Milesi G, Manzoni M, Mazzoni M, Greco A, Tonon G, et al. Molecular spectrum of BRAF, NRAS, and KRAS gene mutations in plasma cell dyscrasias: implication for MEK-ERK pathway activation. Oncotarget. 2015; 6:24205-24217. DOI: 10.18632/oncotarget.4434.

9. Lykke-Andersen S, Tomecki R, Jensen TH, Dziembowski A. The eukaryotic RNA exosome: same scaffold but variable catalytic subunits. RNA Biol. 2011; 8:61-66.

10. Tomecki R, Drazkowska K, Kucinski I, Stodus K, Szczesny RJ, Gruchota J, Owczarek EP, Kalisiak K, Dziembowski A. Multiple myeloma-associated hDIS3 mutations cause perturbations in cellular RNA metabolism and suggest hDIS3 PIN domain as a potential drug target. Nucleic Acids Res. 2014; 42:1270-1290.

11. de Groen FL, Krijgsman O, Tijssen M, Vriend LE, Ylstra B, Hooijberg E, Meijer GA, Steenbergen RD, Carvalho B. Gene-dosage dependent overexpression at the $13 q$ amplicon identifies DIS3 as candidate oncogene in colorectal cancer progression Genes Chromosomes. Cancer. 2014; 53:339-348.

12. Pils D, Tong D, Hager G, Obermayr E, Aust S, Heinze G, Kohl M, Schuster E, Wolf A, Sehouli J, Braicu I, Vergote I, Van Gorp T, et al. A combined blood based gene expression and plasma protein abundance signature for diagnosis of epithelial ovarian cancer-a study of the OVCAD consortium. BMC Cancer. 2013; 13:178.

13. Weißbach S, Langer C, Puppe B, Nedeva T, Bach E, Kull M, Bargou R, Einsele H, Rosenwald A, Knop S, Leich E. The molecular spectrum and clinical impact of DIS3 mutations in multiple myeloma. Br J Haematol. 2015; 169:57-70

14. Segalla S, Pivetti S, Todoerti K, Chudzik MA, Giuliani EC, Lazzaro F, Volta V, Lazarevic D, Musco G, Muzi-Falconi M, Neri A, Biffo S, Tonon G. The ribonuclease DIS3 promotes let-7 miRNA maturation by degrading the pluripotency factor LIN28B mRNA. Nucleic Acids Res. 2015; 43:5182-93.

15. Lorentzen E, Basquin J, Tomecki R, Dziembowski A, Conti E. Structure of the active subunit of the yeast exosome core, Rrp44: diverse modes of substrate recruitment in the RNase II nuclease family. Mol Cell. 2008; 29:717-728.

16. Agnelli L, Mosca L, Fabris S, Lionetti M, Andronache A, Kwee I, Todoerti K, Verdelli D, Battaglia C, Bertoni F, Lambertenghi Deliliers G, Neri A. A SNP microarray and FISH-based procedure to detect allelic imbalances in 
multiple myeloma: an integrated genomics approach reveals a wide gene dosage effect. Genes Chromosomes Cancer. 2009; 48:603-614.

17. Mosca L, Musto P, Todoerti K, Barbieri M, Agnelli L, Fabris S, Tuana G, Lionetti M, Bonaparte E, Sirchia SM, Grieco V, Bianchino G, D'Auria F, et al. Genome-wide analysis of primary plasma cell leukemia identifies recurrent imbalances associated with changes in transcriptional profiles. Am J Hematol. 2013; 88:16-23.

18. Dziembowski A, Lorentzen E, Conti E, Seraphin B. A single subunit, Dis3, is essentially responsible for yeast exosome core activity. Nat Struct Mol Biol. 2007; 14:15-22.

19. Leich E, Weissbach S, Klein HU, Grieb T, Pischimarov J, Stuhmer T, Chatterjee M, Steinbrunn T, Langer C, Eilers M, Knop S, Einsele H, Bargou R, et al. Multiple myeloma is affected by multiple and heterogeneous somatic mutations in adhesion- and receptor tyrosine kinase signaling molecules. Blood Cancer Journal. 2013; 3:e102.

20. Cifola I, Lionetti M, Pinatel E, Todoerti K, Mangano E, Pietrelli A, Fabris S, Mosca L, Simeon V, Petrucci MT, Morabito F, Offidani M, Raimondo FD, et al. Whole-exome sequencing of primary plasma cell leukemia discloses heterogeneous mutational patterns. Oncotarget. 2015; 6:17543-58.

21. Chekanova JA, Gregory BD, Reverdatto SV, Chen H, Kumar R, Hooker T, Yazaki J, Li P, Skiba N, Peng Q, Alonso J, Brukhin V, Grossniklaus U, et al. Genome-wide high-resolution mapping of exosome substrates reveals hidden features in the Arabidopsis transcriptome. Cell. 2007; 131:1340-1353.

22. Houalla R, Devaux F, Fatica A, Kufel J, Barrass D, Torchet C, Tollervey D. Microarray detection of novel nuclear RNA substrates for the exosome. Yeast. 2006; 23:439-454.

23. Kiss DL, Andrulis ED. Genome-wide analysis reveals distinct substrate specificities of Rrp6, Dis3, and core exosome subunits. RNA. 2010; 16:781-791.

24. Eckard SC, Rice GI, Fabre A, Badens C, Gray EE, Hartley JL, Crow YJ, Stetson DB. The SKIV2L RNA exosome limits activation of the RIG-I-like receptors. Nat Immunol. 2014; 15:839-845.

25. Rashid NU, Sperling AS, Bolli N, Wedge DC, Van Loo P, Tai YT, Shammas MA, Fulciniti M, Samur MK, Richardson PG, Magrangeas F, Minvielle S, Futreal PA, et al. Differential and limited expression of mutant alleles in multiple myeloma. Blood. 2014; 124:3110-3117.

26. Musto P, Simeon V, Martorelli MC, Petrucci MT, Cascavilla N, Di Raimondo F, Caravita T, Morabito F, Offidani M, Olivieri A, Benevolo G, Mina R, Guariglia R, et al. Lenalidomide and low-dose dexamethasone for newly diagnosed primary plasma cell leukemia. Leukemia. 2014; 28:222-225.

27. International Myeloma Working G. Criteria for the classification of monoclonal gammopathies, multiple myeloma and related disorders: a report of the International Myeloma Working Group. Br.J.Haematol. 2003; 121:749-757.

28. Durie BG, Salmon SE. A clinical staging system for multiple myeloma. Correlation of measured myeloma cell mass with presenting clinical features, response to treatment, and survival. Cancer. 1975; 36:842-854.

29. Agnelli L, Fabris S, Bicciato S, Basso D, Baldini L, Morabito F, Verdelli D, Todoerti K, Lambertenghi-Deliliers G, Lombardi L, Neri A. Upregulation of translational machinery and distinct genetic subgroups characterise hyperdiploidy in multiple myeloma. $\mathrm{Br}$ J Haematol. 2007; 136:565-573.

30. Todoerti K, Agnelli L, Fabris S, Lionetti M, Tuana G, Mosca L, Lombardi L, Grieco V, Bianchino G, D’Auria F, Statuto T, Mazzoccoli C, De Luca L, et al. Transcriptional characterization of a prospective series of primary plasma cell leukemia revealed signatures associated with tumor progression and poorer outcome. Clin Cancer Res. 2013; 19:3247-3258.

31. Lombardi L, Poretti G, Mattioli M, Fabris S, Agnelli L, Bicciato S, Kwee I, Rinaldi A, Ronchetti D, Verdelli D, Lambertenghi-Deliliers G, Bertoni F, Neri A. Molecular characterization of human multiple myeloma cell lines by integrative genomics: Insights into the biology of the disease. Genes Chromosomes \& Cancer. 2007; 46:226-238.

32. Verdelli D, Nobili L, Todoerti K, Mosca L, Fabris S, D’Anca M, Pellegrino E, Piva R, Inghirami G, Capelli C, Introna $\mathrm{M}$, Baldini L, Chiaramonte $\mathrm{R}$, et al. Molecular events underlying interleukin-6 independence in a subclone of the CMA-03 multiple myeloma cell line. Genes Chromosomes Cancer. 2014; 53:154-167.

33. Fabris S, Agnelli L, Mattioli M, Baldini L, Ronchetti D, Morabito F, Verdelli D, Nobili L, Intini D, Callea V, Stelitano C, Lombardi L, Neri A. Characterization of oncogene dysregulation in multiple myeloma by combined FISH and DNA microarray analyses. Genes Chromosomes Cancer. 2005; 42:117-127.

34. Mattioli M, Agnelli L, Fabris S, Baldini L, Morabito F, Bicciato S, Verdelli D, Intini D, Nobili L, Cro L, Pruneri G, Callea V, Stelitano C, et al. Gene expression profiling of plasma cell dyscrasias reveals molecular patterns associated with distinct IGH translocations in multiple myeloma. Oncogene. 2005; 24:2461-2473.

35. Lionetti M, Fabris S, Cutrona G, Agnelli L, Ciardullo C, Matis S, Ciceri G, Colombo M, Maura F, Mosca L, Gentile M, Recchia AG, Ilariucci F, et al. Highthroughput sequencing for the identification of NOTCH1 mutations in early stage chronic lymphocytic leukaemia: biological and clinical implications. Br J Haematol. 2014; 165:629-639.

36. Tusher VG, Tibshirani R, Chu G. Significance analysis of microarrays applied to the ionizing radiation response. Proc Natl Acad Sci U S A. 2001; 98:5116-5121. 